\title{
An Improved Digital Filtering Method Based on Weighted Average of Input and Output Data
}

\author{
Qiang Zhou ${ }^{1, \text { a }}$, Huali Wu ${ }^{2, \mathrm{~b}}$, Yong $\mathrm{Liu}^{3}$, Junwei Lei ${ }^{2}$ \\ ${ }^{1}$ Department of Scientific Research, Naval Aeronautical and Astronautical University, Yantai, \\ 264001, China \\ ${ }^{2}$ Department of Control, Naval Aeronautical and Astronautical University, Yantai, 264001,China \\ ${ }^{3}$ Department of Navigation, Naval University of Engineering, Wuhan, 430033, China \\ aemail: zhouqiang1024@126.com, bemail: tom_linhuali@126.com
}

Keywords: Digital filter; Low-pass filter; Weighted average; First order

\begin{abstract}
Digitalization of low-pass filter produced from first-order inertia link is carried out firstly in this paper. Then an improved algorithm is proposed to achieve better low-pass characteristics by weighted smoothing the first n-part of the input and output data and using the smoothed data instead of $n-1$ based on the first step. At last, through the detailed numerical simulation analysis, the correctness and validity of the proposed improved filter design method are verified.
\end{abstract}

\section{Introduction}

Digital filter is a digital device that processes signal by a certain algorithm, and it is usually used to inhibit a band of the signal to get a new signal, making the measurement or display more accurate and more reliable [1-3]. Many scholars have proposed many effective algorithms. Such as median digital filtering method to overcome the large pulse limiting [4-5], the average filtering method to suppress the small high-frequency noise [6], and the compound filtering method with the above two method combined [7]. However, at present, the classical filtering methods are more or less to increase the fuzzy information [8]. Therefore, it is difficult to find a filtering method which can remove the signal distortion and noise at the same time. Based on the principle of average filtering, this paper presents a filtering method based on the weighted average of input and output data, which preserves the details of the signal while filtering the noise.

\section{Model Description of Traditional Low-pass Filter}

The traditional low-pass filter is generally designed as the following first-order system, which can be described by differential equation 1as follows [9].

$$
\dot{y}=-\frac{1}{T} y+\frac{1}{T} u
$$

The transfer function can also be described as following formula 2 [10].

$$
\frac{y}{u}=\frac{1}{T s+1}
$$

The following numerical equation 3 is discretized using computer programming.

$$
[y(n)-y(n-1)] / \Delta T=[-y(n-1)+u(n-1)] / T
$$

Where $\Delta T$ is the sampling interval. The formula 3 can be organized as formula 4 .

$$
y(n)=y(n-1)-\frac{\Delta T}{T} y(n-1)+\frac{\Delta T}{T} u(n-1)
$$

\section{Simulation Analysis of Traditional Low-pass Filter}

Set $T=0.1 s$, assuming that the input signal is $u=u_{a}+u_{b}, u_{a}=\sin (5 t), u_{b}=2 \sin \left(10^{5} t\right)$, and $0.2 \sin \left(10^{5} t\right)$ is the noise signal. The performance of the designed filter is verified by comparing 
the signal before the filter with that after the filter [11]. The simulation results are shown from figure1 to figure 3.

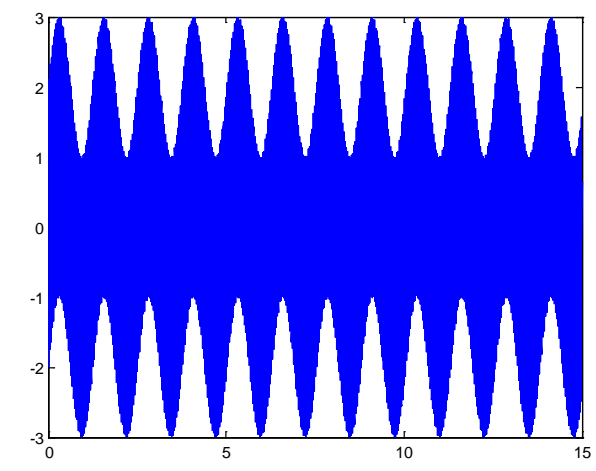

Figure 1 Source signal mixed with noise signal

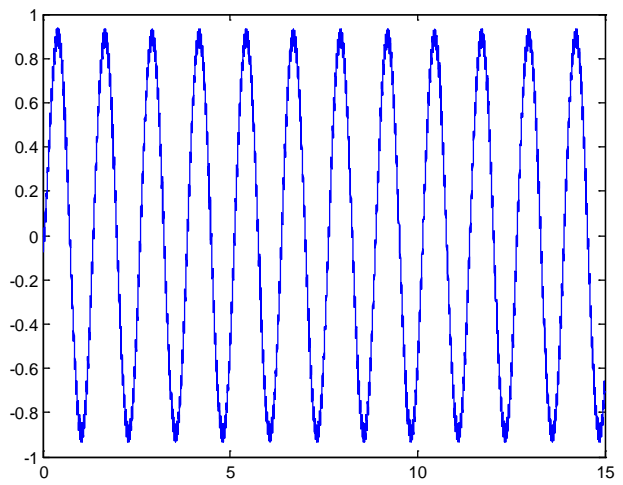

Figure 2 Filtered signal

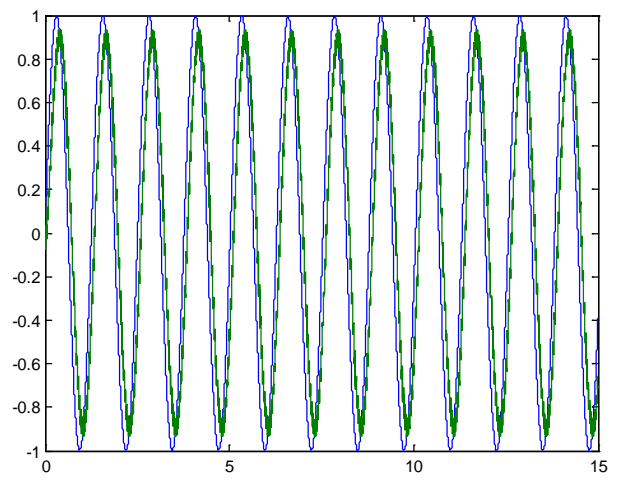

Figure 3 Comparison of filtered signal with useful signal

\section{Improvement Design by Weighted Smoothing}

The following improvement design is proposed as formula 5.

$$
y(n)=\sum_{i=0}^{j} a_{i} y(n-1-i)+\sum_{i=1}^{k} b_{i} \frac{\Delta T}{T} y(n-1-i)-\sum_{i=1}^{l} c_{i} \frac{\Delta T}{T} u(n-1)
$$

And it satisfies the formula 6.

$$
\sum_{i=0}^{l} c_{i}=1, \quad \sum_{i=0}^{l} b_{i}=1, \quad \sum_{i=0}^{j} a_{i}=1
$$

Simulation results are shown from figure 4 to figure 6 . 


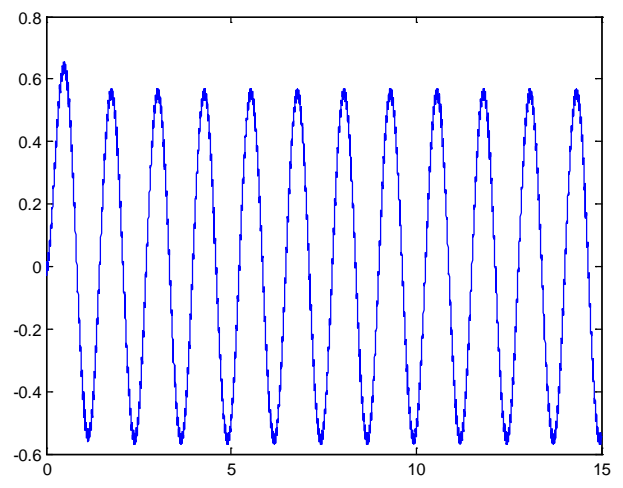

Figure 4 Signal of the improved digital filtering

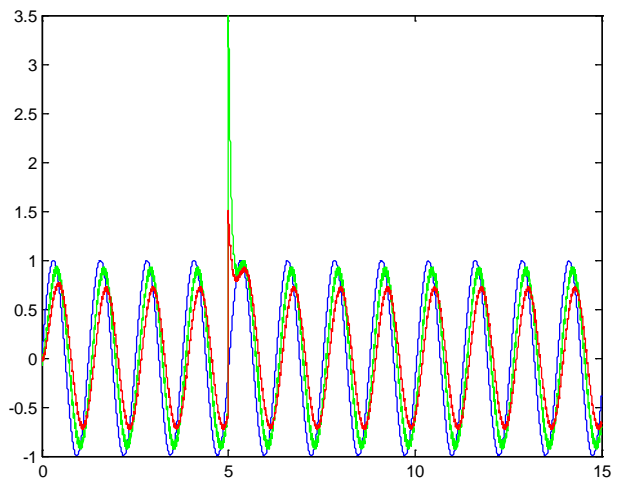

Figure 5 Comparison of the filter signal with the original signal

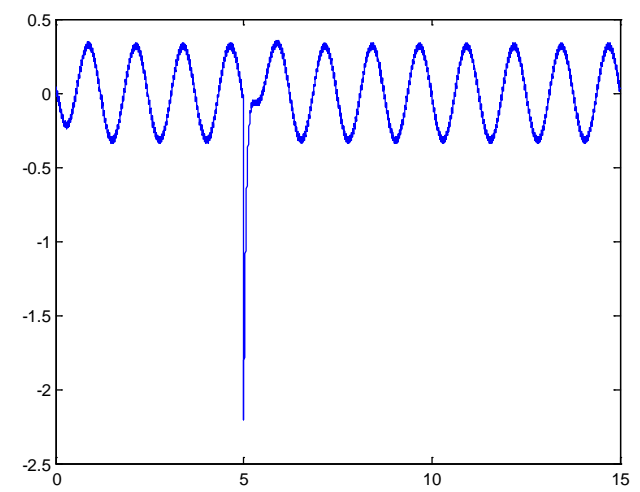

Figure 6 Error Curves of Two Methods

Figure 1 shows the curve of original signal mixed with noise signal, and figure 2 shows the signal from filter designed according the traditional low pass filter, and figure 3 shows the comparison between the useful signal and signal from filter. Figure 4 shows filter result of the improved filter method, Figure 5 shows the difference of the two methods. and figure 5 shows comparison of filter signal with the original signal. And at $5 \mathrm{~s}$, we set a singular point, so the improved method can response to that quickly and error is smaller than the traditional method.

\section{Conclusions}

Low-pass filter is designed based on first-order model in this paper. Then an improved algorithm is proposed by weighed average smoothing of the first n-part of input and output data and using the smoothed data instead of $n-1$. Simulation results show that the improve algorithm has better low-pass performance, which can effectively remove the noise and filter the desired signal. . 


\section{Reference}

[1] Wang Ling, Filter Research and Simulation [J], Industrial Control Computer, 2008 (7): 90-92(in Chinese).

[2] Peng Yongsheng, Wang Taiyong, Low-pass filtering technology in measurement and control system [J], Precision Manufacturing and Automation, 2003 (S1): 108-110(in Chinese).

[3] Zhao Yi, Digital filtering of the moving average and low-pass filter method [J]. Instrument Technology, 2001 (5): 40-40(in Chinese).

[4] A. Eleuteri, A. C. Fisher, D. Groves, C. J. Dewhurst, An Efficient Time-Varying Filter for Detrending and Bandwidth Limiting the Heart Rate Variability Tachogram without Resampling: MATLAB Open-Source Code and Internet Web-Based Implementation[J], Computational \& Mathematical Methods in Medicine, 2012, 2012(2012):259-283.

[5] Chen Jian, Zheng Shaohua, Yu Lun, Improved algorithm for adaptive median filter with multi-threshold based on directional information[J], Journal of Electronic Measurement \& Instrument, 2013, 27(2):156-161.

[6] Guo Wei, Improved Multilevel Non-linear Weighted Mean Median Filter Algorithm [J], Modern Electronics Technique, 2006(19):159-161.

[7] Wen Changbao, Gao Lihong, Fang Jishan, The High-Precision Weighing System Based on the Improved Amplitude-Limiting and Average Filtering Algorithm[J], Chinese Journal of Sensors \& Actuators, 2014, 27(5):649-653.

[8] SX Jia, XH Ren, TW Wang, LF Liang, An improved algorithm of weighted mean filtering[J], Informationization, 2009(16):30-32.

[9] Li Shengqing, Zhou Youqing, Zhu Yinghao, Harmonic current prediction method of integrated power filter based on weighted first-order local theory [J], Proceeding of the CSEE, 2004, 24 (6): 19-23(in Chinese).

[10] ShouLin Sheng, First-order recursive digital filter used in tele-control sysetem [J], Journal of Xian Jiaotong University, 1989(s1):115-120.

[11] Han Lichu et al. MATLAB Electronic Simulation and Application [M]. Beijing: National Defense Industry Press, 2003(in Chinese). 